\title{
Anciennes et nouvelles technologies : métaphores de l'esprit linguistique
}

Alex Boulton

\section{(2) OpenEdition}

1 Journals

\section{Édition électronique}

URL : http://journals.openedition.org/asp/2588

DOI : 10.4000/asp.2588

ISBN : 978-2-8218-0380-0

ISSN : 2108-6354

\section{Éditeur}

Groupe d'étude et de recherche en anglais de spécialité

Édition imprimée

Date de publication : 1 décembre 1999

Pagination : 323-333

ISSN : 1246-8185

\section{Référence électronique}

Alex Boulton, « Anciennes et nouvelles technologies : métaphores de l'esprit linguistique », ASp [En ligne], 23-26 | 1999, mis en ligne le 10 novembre 2011, consulté le 30 avril 2019. URL : http:// journals.openedition.org/asp/2588; DOI : 10.4000/asp.2588

Ce document a été généré automatiquement le 30 avril 2019

Tous droits réservés 


\title{
Anciennes et nouvelles technologies : métaphores de l'esprit linguistique
}

\author{
Alex Boulton
}

\section{Introduction}

1 Le XX ${ }^{e}$ colloque du GERAS avait pour thème un seul mot : réseau. La Lettre du GERAS n ${ }^{\circ} 15$ du 31 décembre 1998 faisait état de l'inquiétude de certains collègues devant une « dérive technologique» du GERAS; il s'agit même peut-être d'une inquiétude devant les nouvelles technologies en général. Ce type d'inquiétude est tout à fait compréhensible les réseaux informatiques sont à la base d'une véritable révolution que nous pouvons difficilement ignorer; il s'agit d'une nouvelle technologie. Tout ce qui est nouveau peut être perçu comme une menace, difficile à comprendre, difficile à intégrer dans une philosophie préexistante, et il nous est difficile de trouver des repères. Ceci nous en dit déjà long sur l'esprit humain: nous réarticulons de nouveaux concepts en termes de concepts déjà connus. On peut aller plus loin: lorsque l'homme se trouve face à de nouvelles informations, il ne peut les raisonner que dans le contexte de toutes les informations déjà présentes dans son esprit : il s'agit d'un travail d'accommodation entre le connu et l'inconnu. Dans un sens très large du terme, il est cette application d'un concept connu à la compréhension ou à l'expression d'un concept nouveau que nous appellerons la métaphore. Citons tout de suite George Lakoff et Mark Johnson, dont l'ouvrage Metaphors We Live By a changé toute notre perception de la métaphore, et qui est toujours aussi pertinent aujourd'hui :

Metaphor is for most people a device of the poetic imagination and the rhetorical flourish - a matter of extraordinary rather than ordinary language. Moreover, metaphor is typically viewed as characteristic of language alone, a matter of words rather than thought or action. For this reason, most people think they can get along perfectly well without metaphor. We have found, on the contrary, that metaphor is pervasive in everyday life, not just in language but in thought and action. Our ordinary conceptual system, in terms of which we both think and act, is fundamentally metaphorical in nature. $(1980: 3)$ 
2 Dans cet article, nous n'aborderons pas directement ces nouvelles technologies; ce qui nous intéresse, c'est le rôle qu'elles jouent en fournissant de nouvelles métaphores pour notre compréhension de l'esprit humain et des fonctions linguistiques humaines, en particulier en ce qui concerne le lexique mental. Nous commencerons par une évaluation de l'importance et de l'omniprésence de la métaphore dans le langage quotidien, avant de passer en revue certains dangers inhérents à une dépendance excessive. Ensuite, nous présenterons quelques métaphores qui ont été appliquées à l'esprit humain, au langage en général, et au lexique mental en particulier. Nous en retiendrons un concept récent et dominant, celui de "réseau », qui sert de point de départ pour un retour aux associations lexicales comme outil d'enquête sur le lexique mental de l'apprenant d'une langue étrangère.

\section{La métaphore au quotidien}

3 La métaphore est une caractéristique universelle et apparemment unique à l'homme. Richard Dawkins (1998) prétend que l'emploi de métaphore serait à l'origine de la croissance explosive de la taille du cerveau humain; d'une manière comparable, Terence Deacon (1997) attribue l'évolution du langage humain à une capacité de fonctionner de manière symbolique: aucune autre espèce, même pas les chimpanzés dans les expériences américaines d'après-guerre, ne fait preuve d'une capacité de représenter un signifié par un signifiant comme nous le faisons chaque instant de chaque jour.

Nous arrivons maintenant au langage quotidien. Selon Lakoff et Johnson (1980), les notions les plus fondamentales à notre pensée sont conceptualisées en termes de métaphores. En majorité, ces métaphores sont évidentes une fois qu'on les remarque : time is money et le temps c'est de l'argent sont même des expressions courantes. Celles-ci, liées à d'autres métaphores en anglais, comme time is a limited resource et time is a valuable commodity, nous fournissent les contextes suivants :

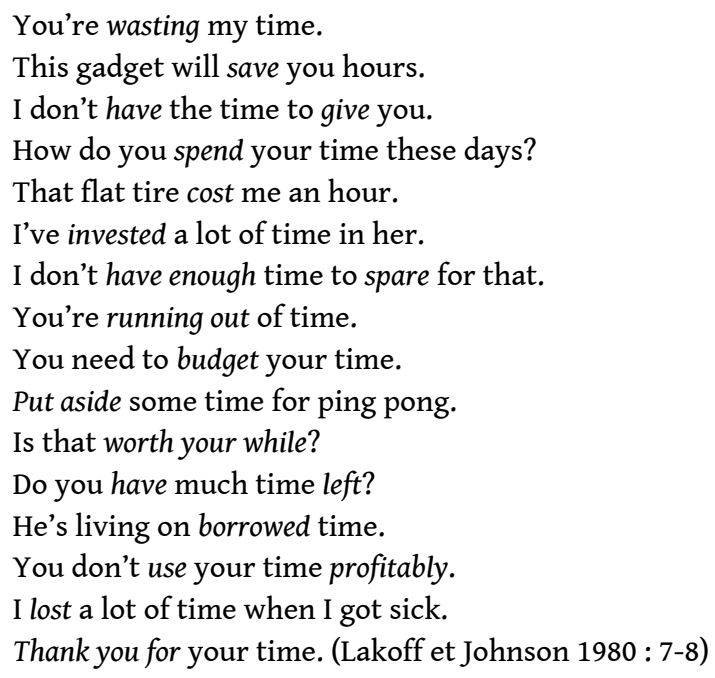

Que l'on en soit conscient ou pas, il y a lieu de supposer que time et money sont intimement liés dans l'esprit de tout locuteur anglophone. Cette métaphore représente bien plus qu'un simple effet de style littéraire; ce sont les concepts qui sont liés, pas seulement les mots. Lorsqu'il s'agit de créer une nouvelle métaphore, c'est-à-dire d'employer ce procédé de façon consciente et délibérée, il est généralement question d'un 
concept cible nouveau et difficile à décrire directement. Avec une bonne métaphore, plusieurs éléments essentiels du nouveau concept ont un équivalent dans un concept source bien connu, où ils doivent être très évidents.

Bien sûr, ce processus n'est pas sans difficulté. La métaphore, tout d'abord, implique plus qu'une simple comparaison. Elle mène à une identification, voire à une fusion entre les deux éléments, qui peuvent par conséquent être confondus. C'est pour cette raison que nous préférons généralement comparer des éléments de deux champs conceptuels bien distincts; bizarrement, la première des deux phrases suivantes est plus facile à comprendre que la deuxième :

His car is a penis.

His car is a lorry.

7 En outre, il est fondamental à la notion même de métaphore que les deux éléments comparés ne soient pas identiques - sinon il ne s'agirait pas d'une métaphore, mais d'une seule et unique chose. Ceci peut être dangereux lorsque la métaphore se fond en analogie, c'est-à-dire l'inférence que, si deux éléments se ressemblent sur certains points importants, il est probable qu'ils se ressembleront sur d'autres. Par exemple, en ce qui concerne l'apprentissage des langues, nous ne devrions pas nous étonner que l'apprenant essaie d'analyser une langue étrangère en fonction de sa langue maternelle : la L1 sert de métaphore pour la L2. Ce type de raisonnement peut offrir de nouvelles perspectives: l'apprenant apprend plus vite que s'il commençait ab initio, mais cette approche peut également mener à certaines erreurs bien connues.

Chaque métaphore est ainsi capable de nous aider dans notre compréhension d'un nouveau concept, mais chacune aura nécessairement ses faiblesses en même temps.

\section{Métaphores linguistiques}

9 La conceptualisation de l'esprit humain n'est pas facile. McCarthy (1990 : 34) rappelle que notre compréhension du langage et du lexique mental s'est développée avec l'évolution de nombreuses métaphores, à commencer par un verger, un chemin ou un magasin, une bibliothèque, un dictionnaire ou une encyclopédie, jusqu'au central téléphonique, entre autres. Toutes ces métaphores ont leurs propres problèmes - en particulier, elles suggèrent un endroit fixe où sont placées les informations différentes; les informations sont placées soit en désordre, soit selon un seul critère, par exemple en ordre alphabétique ; l'utilisateur se promène dedans pour trouver ce dont il a besoin, ou bien il $\mathrm{y}$ accède grâce à un index, fichier, catalogue ou autre système central ; cet utilisateur est une sorte d'« homuncule » qui a son propre esprit... qui a son propre homuncule qui a son propre esprit, etc. Ces métaphores ont été abandonnées pour toutes ces raisons, et parce qu'elles ne reflètent pas la nature dynamique de l'esprit humain.

D'autres métaphores attirent l'attention sur différents aspects de l'esprit: l'esprit est comme une éponge, dans la mesure où il peut absorber et restituer des informations; mais cela ne dit rien sur la structure de l'esprit ni sur les mécanismes responsables de ces actes (Tooby \& Cosmides 1992). L'esprit est comme un canif de l'armée suisse, dans la mesure où il dispose de différents outils (modules) afin d'accomplir différentes tâches (Mithen 1996). Le problème de ce type de métaphore c'est que l'esprit apparait comme 
figé dès la naissance, sans qu'aucune place soit laissée au développement de l'esprit individuel.

11 L'arrivée des « nouvelles technologies » a apporté de nouvelles possibilités de métaphore, qui méritent l'attention. Dès son apparition et jusque dans les années 1980, la métaphore de l'ordinateur numérique dominait. Les parallèles entre l'esprit et l'ordinateur sont assez évidents, mais ce qui est évident n'est pas forcément juste. Depuis, des insuffisances très importantes ont été décelées, et la métaphore est tombée en désuétude, bien qu'elle domine toujours dans de nombreuses autres situations (Aitchison 1994 : 152).

À la différence de l'ordinateur, où le signal voyage presque à la vitesse de la lumière, la transmission du signal dans le cerveau est en effet très lente; il semble clair que de nombreux éléments de toutes sortes sont traités simultanément en parallèle dans l'esprit, et non pas en série comme pour un ordinateur. Autre problème de l'ordinateur comme métaphore de l'esprit : il fonctionne avec un nombre fini d'états possibles, et ne peut donc pas rendre compte de la créativité et de l'imprévisibilité de l'esprit humain. L'ordinateur a un fonctionnement numérique binaire bien adapté à certains types de problème $(1 / 0$; A/B; OUI/NON; VRAI/FAUX...), tandis que nous avons tendance à un fonctionnement analogue (PLUS/MoINS, des nuances de GRIS...). Effectivement, plusieurs difficultés rencontrées dans les modèles psycholinguistiques traditionnels résultent d'une conception binaire, qui ne permet pas de position intermédiaire.

13 Ceci nous amène à l'informatique, une métaphore venue de la psychologie cognitive. L'esprit y est représenté comme un système d'entrée, de stockage, de traitement et de sortie d'informations. Cette métaphore est très puissante, et nous fournit la majeure partie de la terminologie employée actuellement dans ce domaine. Les principaux inconvénients sont souvent le résultat d'une certaine mésentente : en tant que métaphore de l'esprit, l'informatique n'a rien à voir avec l'ordinateur. Si l'on compare toujours le cerveau ou l'esprit à un ordinateur, c'est à cause de ce que l'un et l'autre font, pas à cause de ce qu'ils sont.

Les philosophes ont longtemps débattu de la distinction entre cerveau et esprit. Il serait tentant d'y voir une correspondance entre les éléments suivants (voir fig. 1).

Figure 1

\begin{tabular}{|l|l|l|}
\hline cerveau & $>$ & esprit \\
\hline ordinateur & $>$ & logiciels \\
\hline neurologie & $>$ & psychologie \\
\hline matériel informatique & $>$ & programmes informatiques \\
\hline
\end{tabular}

Cependant, la séparation entre cerveau et esprit relève d'une controverse largement dépassée: la pensée change l'architecture même du cerveau, et les changements de l'architecture permettent la pensée. Les deux formulations sont justes, et il est ainsi impossible de dissocier l'esprit du cerveau. Cette découverte a permis de se référer au cerveau pour la dernière génération de métaphores évoquant l'esprit - développement déjà très fructueux, qui commence à concurrencer la métaphore de l'informatique. 


\section{mettre en évidence à travers quelques chiffres :}

1. un neurone peut se déclencher jusqu'à cinquante mille fois par minute

2. le cerveau dispose d'une centaine de milliards $\left(10^{11}\right)$ de neurones

3. il existe cent mille milliards $\left(10^{14}\right)$ de connexions entre les neurones

4. le tout est assuré par des millions de kilomètres de fibres nerveuses

Pour mettre cela en perspective, il a été estimé que le nombre d'états possibles d'un cerveau humain dépasse le nombre de particules élémentaires dans l'univers (Ramachandran et Blakeslee 1998 : 8).

18 Cette vision de la complexité est renforcée par un élément clé de cette métaphore : le cerveau et l'esprit sont tous les deux des produits de l'évolution selon la théorie néodarwinienne. Cette perspective de la psychologie évolutionnaire (voir, par exemple, Barkow et al. 1992) fait apparaître un esprit «bricolé » au cours du temps, comme (pour prendre une autre métaphore) une ville qui se construit au cours des siècles, maison par maison, rue par rue, avec une restructuration continue. Le résultat est d'une complexité phénoménale, et le tout fonctionne en parallèle, puisque c'est « dans la complexité [que] se trouve la compétence pour faire face à la diversité » (Besner \& Johnston 1989 : 312). Nous apprenons ainsi que la quête sacrée d'une description complète d'une langue est impossible ; plus important, la simplicité n'est plus la condition sine qua non d'une théorie psycholinguistique.

\section{Réseaux... informatiques et neurologiques}

19 Grâce au développement de l'Internet, le terme "réseau » est un mot à la mode actuellement dans plusieurs domaines, dont la neurologie, l'informatique... et la psychologie. Dans ces trois cas, les noyaux sont représentés par des neurones, par des ordinateurs ou par des entrées d'informations; ces noyaux sont interconnectés pour donner naissance à la structure qui est respectivement le cerveau, l'Internet, ou l'esprit. Mais, tandis qu'il est possible de visualiser le réseau Internet en deux dimensions, il est quasiment impossible de faire de même avec le cerveau, ou encore avec l'esprit.

On peut facilement concevoir l'esprit comme un réseau multidimensionnel d'informations, qui permet l'apprentissage, le stockage, le traitement et la récupération de ces informations. Les liens dépendent du fait que les entrées partagent certains traits. On peut partir du principe que toute entrée qui contient un trait donné sera liée à toute autre entrée contenant le même trait. Ainsi, les informations ne sont pas organisées en une simple liste, mais simultanément selon des critères multiples. Les expériences de rappel libre révèlent que presque tout ce qui peut servir à lier deux entrées peut être déployé (Greene 1987) ; les informations contenues dans les entrées sont donc de natures très diverses. En ce qui concerne le lexique mental, on peut concevoir l'existence de liens basés sur la phonologie, la graphie, la morphologie, l'étymologie, la grammaire, la sémantique, la traduction, etc. (Boulton 1998).

21 Le terme "réseau » était employé en psychologie bien avant le développement de l'Internet. Cela dit, puisque l'Internet et le cerveau servent actuellement de métaphores de l'esprit, et puisque les deux constituent deux visions très différentes d'un réseau, il est important de les distinguer. Quelle métaphore de l'esprit est plus appropriée - la 
nouvelle technologie de l'Internet, développée au début des années 1970, ou la technologie du cerveau humain, vieille de peut-être 100000 ans?

Dans le contexte d'Internet, le réseau est composé de liens changeants - même virtuels entre d'innombrables ordinateurs; dans le contexte du cerveau, il représente les connexions synaptiques entre les neurones. Dans l'esprit, lorsqu'une entrée d'information en active une autre, le message est envoyé à travers une ou plusieurs connexions dans le réseau. Selon les différentes métaphores, une connexion pourrait être un chemin concret et durable entre deux entrées (comme les synapses et axones du cerveau), ou bien il pourrait être une "adresse » stockée à l'intérieur d'une entrée (comme sur Internet). L'envoi d'un signal est donc le résultat d'une instruction, soit « suivez chemin X» (métaphore neurologique) soit «trouvez adresse X» (métaphore informatique).

Diverses fonctions psycholinguistiques ont été modélisées sur ordinateur ; il est presque ironique que le résultat ressemble beaucoup plus aux réseaux neurologiques qu'au réseau Internet. Brièvement, dans ces modèles, chaque entrée est équipée de détecteurs et/ou et de transmetteurs capables de recevoir et/ou d'émettre un signal. En récupération réceptive ou productive, l'entrée avec les détecteurs les plus fortement activés dominera, et supprimera ainsi toute activité dans les autres entrées, et à ce moment-là, l'accès aura lieu.

Sejnowski et Rosenberg (1987) nous ont fourni une des premières modélisations explicites de ce type de réseau, le programme NETTALK, où le système est programmé afin de découvrir la prononciation d'un mot anglais à partir de normes phonotactiques. Le réseau est simplement exposé à des données, et ne reçoit aucune règle pour l'aider - à vrai dire, le système ne saurait pas quoi faire d'une règle s'il en disposait. Tout comme le cerveau humain, ce type de modèle dit "connexionniste » fonctionne à base de régularités et d'irrégularités perçues, de traitement statistique, de probabilité, et de tendances générales, encore truffée d'exceptions, de flou, de redondance... et non pas à partir de règles formelles.

Si nous pouvons nous permettre une évidence : un ordinateur n'est qu'un ordinateur ; deux ordinateurs reliés ne sont que deux ordinateurs reliés. C'est-à-dire, ils peuvent fonctionner isolément, ou ensemble. Cette évidence est importante parce qu'elle contraste avec le cas des entrées psychologiques, leurs équivalents métaphoriques. Dans l'esprit humain, le stockage durable de toute information dépend de ses liens avec d'autres informations : le système doit sa puissance aux connexions et à la structure du réseau. Pour cette raison, la « compétence » est plus que la somme des connaissances des données individuelles. Une entrée, comme un neurone, se définit par rapport à ses interconnexions; comme un neurone, elle ne peut pas exister toute seule :

Un neurone [...] ne peut vivre, fonctionner, exister, que s'il a pu créer des réseaux ou des circuits avec des congénères. De la qualité de ces réalisations découle le degré des performances du cerveau humain. (Robert 1994 : 52)

Toutes les connexions dans l'esprit ne sont pas identiques. Comme les connexions neurologiques, ces liens psychologiques peuvent être :

- directs ou indirects (il n'y a pas d'équivalent d'un serveur web qui sert d'intermédiaire, les connexions s'effectuant directement d'une entrée à une autre, ou à travers d'autres entrées)

- plus ou moins forts

- plus ou moins nombreux

- qualitativement différents 
- unidirectionnels ou bidirectionnels

- facilitateurs ou inhibiteurs.

La facilité d'accès à une information dépend de la quantité et de la qualité de ses connexions. Les données les mieux connues sont celles qui sont le mieux intégrées dans l'esprit, qui disposent du plus grand nombre de connexions et des plus fortes.

Certaines des entrées L1 sont liées directement, mais toutes le sont indirectement, et ce sont ces liens qui organisent le stock lexical. Paul Meara, dans ses Network Structures and Vocabulary Acquisition in a Foreign Language (1992), a recours à des théories mathématiques pour rendre cette complexité plus compréhensible. Prenons une autre métaphore, celle des États-Unis, avec ses quelque 250 millions d'habitants. Si nous supposons que chaque individu connaît mille personnes, la probabilité que deux personnes (A et B) désignées au hasard se connaissent est une sur 100 000. En revanche, il y a une chance sur 100 que ces deux personnes aient un ami commun (A connaît X, qui connaît B). Et la probabilité qu'elles soient liées par une seule maille supplémentaire dans la chaîne (A connaît $X$, qui connaît $Y$, qui connaît B) dépasse 99 chances sur 100. C'est-à-dire, dans un réseau de 250 millions d'éléments, et avec une valence de 1000 connexions, il est probable à $99 \%$ que deux éléments soient liés par trois connexions tout au plus. Si on estime à 50000 le nombre d'unités lexicales connues par un locuteur (Nation 1990), et que chacune dispose d'une vingtaine de connexions, alors trois étapes seulement sépareraient deux entrées lexicales en moyenne.

\section{Associations}

En examinant différentes implications des métaphores de réseau informatique et neurologique, nous avons presque accidentellement décrit un modèle du lexique mental, basé sur un réseau d'entrées et de connexions (voir Boulton 1998 pour une analyse plus complète). Un des avantages de ce type de modèle est qu'il fait un lien explicite entre, d'un côté, les entrées dans un réseau de connexions (psychologiques) du modèle théorique, et de l'autre, les unités lexicales dans un réseau d'associations (de comportement) que l'on peut examiner directement dans des situations contrôlées. Alors, si les associations lexicales ont été très étudiées chez le locuteur natif, il n'en est pas de même pour l'apprenant. Là, il n'existe qu'une poignée de publications, datant souvent des années 1960 et 1970, la plupart d'entre elles cherchant simplement à démontrer que les associations L2 sont fondamentalement différentes de celles du natif monolingue. Depuis, cette forme de recherche a été largement abandonnée, sans raison évidente.

Afin d'étudier les réseaux d'associations lexicales, nous avons récemment conçu une série d'expériences portant sur les associations lexicales chez les apprenants français de l'anglais L2 (Boulton, 1998). Nous avons pu montrer en même temps la grande souplesse de ce type d'expérience: nous n'avons pas demandé des associations libres, préférant présenter des paires de mots ; les sujets devaient réfléchir à l'association entre chaque paire. L'intérêt de cette conception des associations "inverses " - où le stimulus et la réponse sont fournis tous les deux - est que la situation est beaucoup plus contrôlée. Plus tard, nous avons testé le rappel en présentant un seul mot de chaque paire : les sujets devaient donc retrouver l'autre mot qui l'accompagnait, ainsi que leurs propres associations. Brièvement, les résultats soutiennent un modèle comme celui que nous venons d'esquisser, avec des associations très diverses, ainsi que l'hypothèse qu'il existe 
des liens directs entre les unités lexicales de langues différentes. Nous avions par ailleurs divisé les sujets en quatre groupes avec différentes combinaisons linguistiques du stimulus et de la réponse; ceci a permis de comparer les apprenants avec eux-mêmes dans différentes situations linguistiques. Bien qu'il existe des différences quantitatives selon la situation, elles ne semblent pas refléter des différences qualitatives entre les types d'associations dans les différentes situations. L'apprenant ressemble au natif; les associations interlinguistiques ressemblent aux associations intralinguistiques.

D'autres expériences sont en cours. D'abord, en continuant avec des associations «inverses» nous avons sélectionné pour chaque stimulus trois réponses parmi les normes des locuteurs natifs. Les sujets ont comme tâche de classer ces réponses dans l'ordre de l'intimité de leur association avec le stimulus. S'ils ne réussissent pas dans l'ensemble, cela soutiendra l'opinion courante que les associations des apprenants sont très différentes de celles des natifs à cause d'une compétence lexicale fondamentalement différente. Par contre, si les sujets réussissent, on pourra penser que les différences apparentes lors des expériences classiques ne révèlent que des préférences différentes de la part des apprenants, mais non pas une incapacité linguistique.

Ensuite, une expérience d'association libre plus traditionnelle, qui utilise une liste de mots pour laquelle il existe déjà des données pour des populations anglophones et (avec une traduction de la liste) des populations francophones; la version française a également été utilisée auprès d'un groupe d'apprenants anglais du français L2. Ces expériences complètent le paradigme $\mathrm{L} 2$, et une première analyse révèle des réponses beaucoup plus homogènes que d'autres ne laisseraient entendre, et beaucoup plus semblables aux normes des locuteurs natifs. Finalement, ces mêmes étudiants ont fait la même expérience avec cette fois la traduction française de la liste; en comparant les réponses anglaises et françaises, il sera possible de tester l'importance d'une simple traduction entre les unités des deux langues. Une chose qui apparaît clairement est l'influence de l'interculturel, de la culture internationale pour l'organisation des nombreux mots anglais connus aux étudiants dans des contextes linguistiques français (il ne s'agit pas d'emprunts proprement dits), notamment dans les domaines de l'informatique, du sport, des loisirs... (Boulton 1999).

33 Toutes ces expériences ont été élaborées dans le contexte du modèle théorique rendu possible par la métaphore des réseaux.

\section{Conclusions}

Les métaphores sont essentielles pour une compréhension facile de nouveaux concepts. Elles peuvent nous aider à l'élaboration de nouveaux modèles, et fournir de nouvelles pistes quant aux moyens de mettre ces modèles à l'épreuve. Toutefois, la dépendance aveugle est dangereuse. Malgré leur omniprésence et leur très grande utilité, les nouvelles technologies ont leurs propres limites, il ne s'agit pas d'une panacée dans la didactique des langues étrangères; elles ne fournissent pas non plus de métaphores parfaites de l'esprit humain. En fait, l'Internet ajoute peu d'éléments qui n'étaient pas déjà présents dans la métaphore du central téléphonique. Ainsi, le cerveau nous fournit une métaphore plus apte à rendre compte de la nature de l'esprit tel que nous l'avons décrit. Pour conclure avec un exemple : selon des théories récentes, le cerveau humain crée des modèles du monde à partir de petites bribes d'informations perçues et traitées, et nous fonctionnons par rapport à ce monde « virtuel ». En outre, selon Dawkins (1998), 
le cerveau se sert de toutes les idées que nous avons afin de créer un « soi virtuel ». Mais il y a une grande différence entre le cerveau et l'Internet : le cerveau a donné naissance à l'esprit humain, qui est le résultat du "hasard" de l'évolution; il serait par contre douteux de prétendre que le réseau Internet puisse accidentellement atteindre une « conscience » ou un « soi » lorsqu'il dépassera une taille critique.

\section{BIBLIOGRAPHIE}

Aitchison, Jean. 1994. Words in the Mind: An Introduction to the Mental Lexicon. 2nd éd. Oxford : Basil Blackwell.

Barkow, J.H., L. Cosmides \& J. Tooby (dir.). 1992. The Adapted Mind: Evolutionary Psychology and the Generation of Culture. Oxford: Oxford University Press.

Besner, D. \& J.C. Johnston. 1989. «Reading and the mental lexicon: On the uptake of visual information ». In W.D. Marslen-Wilson (dir.), Lexical Representation and Process. Cambridge MA : MIT Press, 291-316.

Boulton, Alex. 1998. « Le lexique mental L2 : connexions et associations ». Thèse de doctorat, Université de Nancy 2.

Boulton, Alex. 1999. « Associations lexicales interculturelles ». Communication au $27^{\mathrm{e}}$ Congrès UPLEGESS, Évry.

Dawkins, Richard. 1998. Unweaving the Rainbow. Harmondsworth : Penguin.

Deacon, Terence W. 1997. The Symbolic Species: the Co-evolution of Language and the Human Brain. Londres : Allen Lane/Penguin Books.

Greene, J. 1987. Memory, Thinking and Language. Londres : Methuen.

Lakoff, George \& Mark Johnson. 1980. Metaphors We Live By. Chicago, IL : University of Chicago Press.

McCarthy, Michael J. 1990. Vocabulary. Oxford : Oxford University Press.

Meara, Paul. 1992. « Network structures and vocabulary acquisition in a foreign language ». In P.J.L. Arnaud \& H. Béjoint (dir.), Vocabulary and Applied Linguistics. Londres : Macmillan, 62-70.

Mithen, Steven. 1996. The Prehistory of the Mind. Londres : Thames \& Hudson/Phoenix.

Nation, I.S.P. 1990. Teaching and Learning Vocabulary. Rowley, MA : Newbury House.

Ramachandram, V.S. \& S. Blakeslee. 1998. Phantoms in the Brain: Human Nature and the Architecture of the Mind. Londres : Fourth Estate.

Robert, J-M. 1994. Le cerveau. Paris : Flammarion.

Sejnowski, T.J. \& C.R. Rosenberg. 1987. «Parallel networks that learn to pronounce English text ». Complex Systems 1, 145-168. 
Tooby, J. \& L. Cosmides. 1992. «The psychological foundations of culture ». In J.H. Barkow, L. Cosmides \& J. Tooby (dir.), The Adapted Mind: Evolutionary Psychology and the Generation of Culture. Oxford : Oxford University Press, 19-136.

\section{RÉSUMÉS}

L'enseignant de langues étrangères (L2) néglige souvent les connaissances psycholinguistiques, sous prétexte que le "module langue» est très complexe et difficile à cerner de façon compréhensible. Pour cette raison, de la civilisation grecque classique jusqu'à nos jours, l'esprit humain en général et nos fonctions linguistiques en particulier ont été imaginés à l'aide de métaphores. Nous commençons par une brève étude de la nature et du rôle de la métaphore dans le langage et dans la pensée, avant de passer en revue les différentes métaphores employées pour décrire nos fonctions psycholinguistiques, leurs avantages et désavantages relatifs, et ce que chacune apporte à notre compréhension de l'esprit linguistique de l'apprenant L2. Finalement, nous comparons les notions de « réseau » dans les métaphores de l'informatique et du cerveau et leur contribution aux modèles actuels des associations lexicales interlinguistiques.

The foreign language (L2) teacher is often ignorant of relevant psycholinguistic research, complaining that the "language module" is highly complex and difficult to understand. For this reason, from classical Greek civilisation to the present day, the human mind in general and our linguistic functions in particular have been described in terms of metaphors. We begin by outlining the nature and role of metaphor in language and thought, before moving on to an analysis of various metaphors used to describe psycholinguistic functions, their advantages and disadvantages, and what each has brought to our understanding of the linguistic mind of an L2 learner. Finally, we compare the different notions of "network" in metaphors of information processing and the human brain and their contribution to current models of interlingual lexical associations.

\section{INDEX}

Mots-clés : association lexicale, L2, lexique mental, métaphore (linguistique), réseau lexical Keywords : lexical network, mental lexicon, metaphor (linguistic), word association

\section{AUTEUR}

\section{ALEX BOULTON}

Alex Boulton est maître de conférences associé à l'Université Henri Poincaré, Nancy 1. Il a soutenu sa thèse intitulée « Le lexique mental L2 : connexions et associations » en 1998 à l'Université Nancy 2. Ses recherches portent sur des aspects psycholinguistiques de l'apprentissage du vocabulaire L2, le lexique mental d'un apprenant L2 et, plus précisément, la manière dont les lexiques des deux langues coexistent dans un même esprit. Alex.Boulton@univnancy2.fr 